\title{
Corazón roto. ¿cuál es la causa?
}

\author{
What broke my patient's heart?
}

Carmen S López Seijas, Henrique Villena García del Real, Carmen Seijas Rodríguez, Carmen Varela Donoso.
Servicio de Urgencias. Complexo Hospitalario Universitario de Santiago de Compostela (CHUS). SERGAS. Santiago de Compostela

\begin{abstract}
Resumen
El síndrome Tako-Tsubo, o de discinesia apical transitoria (SDAT), es una miocardiopatía aguda y reversible, capaz de producir los mismos síntomas que un infarto agudo de miocardio (IAM), con elevación de biomarcadores y alteraciones electrocardiográficas isquémicas pero con ausencia de lesiones en la coronariografía y una característica discinesia anteroapical del ventrículo izquierdo que se normaliza en pocos días. Es una entidad poco frecuente, que afecta en torno a un $1 \%$ de todos los pacientes con sospecha de síndrome coronario agudo, siendo fundamental realizar un diagnóstico diferencial con el mismo, ya que de ello va a depender el adecuado tratamiento, así como el pronóstico del paciente. Afecta con mayor frecuencia a mujeres postmenopáusicas, con escasos factores de riesgo cardiovascular y tras un desencadenante de estrés emocional o físico.

Presentamos un caso clínico en una paciente de 75 años que presenta un curso clínico típico y cumple los criterios diagnósticos de la enfermedad. Palabras clave. Síndrome de discinesia transitoria del izquierdo. Cardiomiopatía de tako-tsubo. Síndrome de ballooning
\end{abstract}

\section{Introducción}

El síndrome de Tako-Tsubo o discinesia apical transitoria (SDAT) fue descrito por primera vez en Japón en 1990 por Sato et al, pero no se conoce como entidad clínica hasta el año 2011, tras la publicación de una serie de 88 casos $^{1}$. Se caracteriza por la hipocinesia 0 acinesia apical transitoria del ventrículo izquierdo, que presenta un abombamiento apical extenso similar al contorno de una vasija utilizada tradicionalmente en Japón para la captura de pulpos llamada "takotsubo". Su etiopatogenia está todavía por definir. Es una entidad poco frecuente, que afecta en torno a un $1 \%$ de todos los pacientes con sospecha de síndrome coronario agudo ${ }^{1}$, siendo fundamental realizar un diagnóstico diferencial con el mismo, ya que de ello va a depender el adecuado tratamiento, así como el pronóstico del paciente.

Presentamos un caso clínico en una paciente de 75 años que presenta un curso clínico típico y cumple los criterios diagnósticos de la enfermedad.

\section{Caso clínico}

Mujer de 75 años, diagnosticada de trastorno mixto ansioso depresivo en tratamiento farmacológico, sin otros antecedentes de interés. Acude a nuestro centro tras presentar dos horas antes de su asistencia, durante el funeral de su esposo, un cuadro de dolor torácico irradiado a cuello, de inicio brusco y de menos de media hora de duración, acompañado de intenso cortejo vegetativo. Posteriormente refiere otro episodio de las mismas características pero de mayor intensidad, que persiste a su llegada.

\begin{abstract}
Tako-Tsubo syndrome, also known as transient apical ballooning syndrome, is an acute and reversible cardiomyopathy. It presents the same symptoms as an acute myocardial infarction, including the elevation of myocardial enzymes and ischemic electrocardiographic changes. However, no significant blockages will be found in the coronary angiography and there is a typical antero-apical dyskinesia of the left heart ventricle that improves in a few days. This entity is rare, affecting approximately $1 \%$ of all patients with suspected acute coronary syndrome. Nevertheless, it is essential to consider it in the presence of a cardiomyopathy, since its diagnosis will change the treatment and the prognosis for the patient. The typical case is a postmenopausal woman, with few cardiovascular risk factors, who has suffered from a physical or emotional stress.

We present here a case report of a 75 years old female patient who had a typical clinical presentation of the disease, fulfilling the diagnostic criteria for the Tako-Tsubo syndrome.
\end{abstract}

Key words. Transient left ventricular dyskinesia. Tako-Tsubo cardiomyopathy. Ballooning syndrome

Figura 1. ECG inicial: imagen de infarto agudo anterior evolucionado

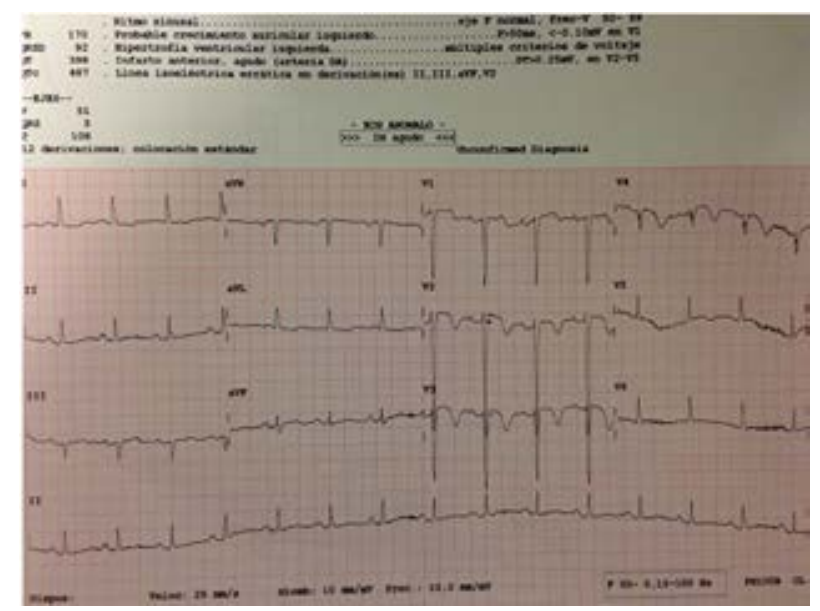

En el electrocardiograma inicial se objetiva una imagen de infarto agudo de miocardio anterior evolucionado: elevación del ST y onda T negativas en cara anterolateral (Figura 1). Se solicita una primera determinación de TPN I siendo esta de $1.2 \mathrm{ng} / \mathrm{dl}$. Se realiza un ecocardiograma en el que se aprecia una marcada acinesia de segmentos apicales, con disfunción ventricular severa (VTD $64 \mathrm{ml} \mathrm{y} \mathrm{VTS} 40 \mathrm{ml}$ con FEVI 35\% por Simpson en apical 4 cámaras).

Ante estos hallazgos se realiza una coronariografía urgente que no evidencia lesiones significativas. Una cardio-RM no mostró focos de miocarditis y la determinación de catecolaminas fue normal. A las 4 semanas se procedió al alta con normalización del ECG y recuperación de función sistólica en el ecocardiograma. Se estableció el diagnóstico de síndrome de Tako-Tsubo según los criterios la clínica Mayo. 
Figura 2. Arte tradicional de pesca japonés para pescar pulpos. Ventriculografía: acinesia de segmentos apicales e hipercontractilidad de segmentos basales causando balonización del ventrículo en sístole
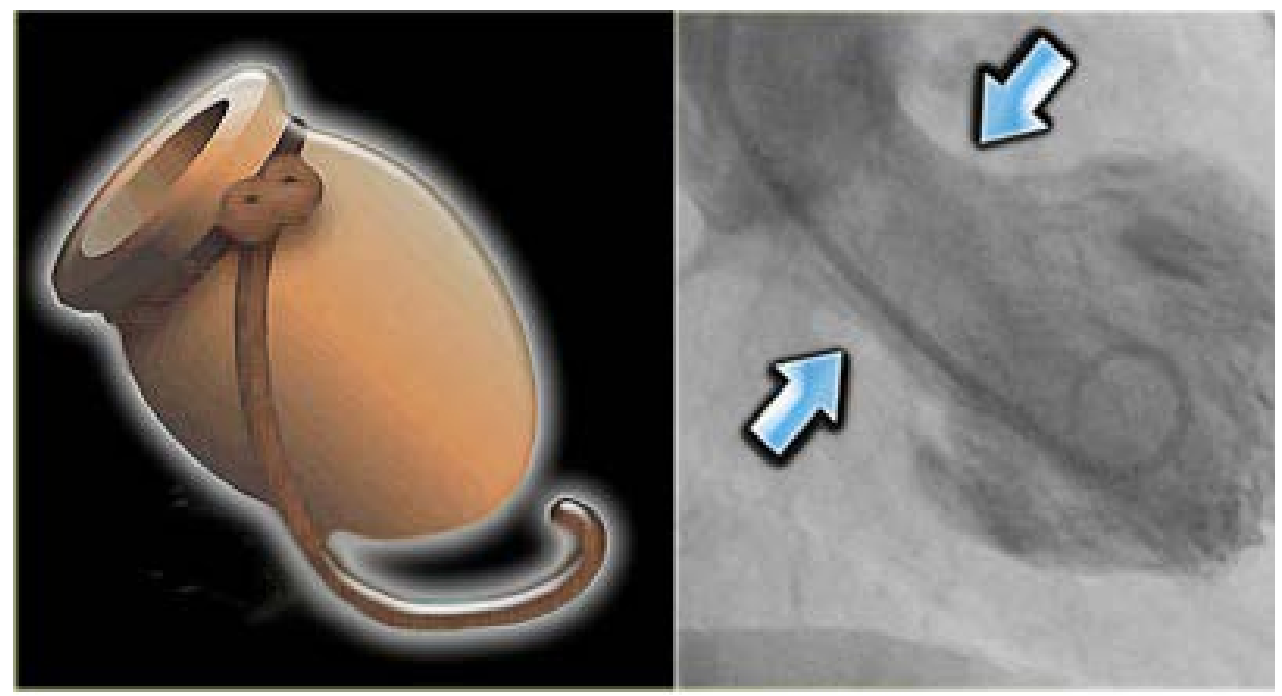

\section{Discusión}

El síndrome Tako-Tsubo, o de discinesia apical transitoria (SDAT), "apical ballooning" o "Síndrome del corazón roto" es una miocardiopatía aguda y reversible, capaz de producir los mismos síntomas que un infarto agudo de miocardio (IAM), con elevación de biomarcadores y alteraciones electrocardiográficas isquémicas.

Es una patología infrecuente, suponiendo sólo el 0,5-1\% de los pacientes con sospecha de Síndrome coronario agudo (SCA $)^{3,5}$. En aproximadamente un $80 \%$ de los casos afecta a mujeres postmenopáusicas, con escasos factores de riesgo cardiovascular y tras un desencadenante de estrés emocional o físico, siendo el estrés emocional severo el desencadenante más común. Se han descrito casos en todas las razas. Su mortalidad es muy baja, menor del 1\%, comparada con la producida por el IAM¹.

EI SDAT se caracteriza por dolor precordial de características anginosas, cambios electrocardiográficos transitorios (en un 90 $\%$ de los casos se evidencia una elevación del segmento ST en cara anterior $\left.{ }^{6,7}\right)$, elevación de marcadores de necrosis miocárdica (en aproximadamente un $50 \%$ de los casos y menor de lo esperado con respecto a los cambios electrocardiográficos'?), ausencia de obstrucción coronaria en la coronariografía y una característica discinesia anteroapical del ventrículo izquierdo objetivable en ecogardiograma que se normaliza en pocos días. Incialmente presenta más complicaciones que el IAM (arritmias ventriculares, shock cardiogénico, edema agudo de pulmón), aunque el pronóstico es mejor a corto y medio plazo ${ }^{1,2}$. Es fundamental realizar un diagnóstico diferencial de otras causas de disfunción transitoria de ventrículo izquierdo como son: feocromocitoma, hemorragia subaracnoidea, secundaria a consumo de tóxicos (cocaína), miocarditis?

Su etiopatogenia, a fecha de hoy, está por definir. Entre los mecanismos etiológicos propuestos se incluyen el vasoespasmo de las arterias coronarias, los trastornos de la microvascularización cardíaca y la disfunción miocárdica secundaria a catecolaminas, entre otras.

En 2008, investigadores de la clínica Mayo establecieron 4 criterios diagnósticos que son los actualmente más utilizados (Tabla 1), debiendo cumplirse los cuatro para establecer el diagnóstico3.

Tabla 1. Criterios diagnósticos de Síndrome de Tako-Tsubo

Alteraciones transitorias de la contractilidad ventricular izquierda (discinesia, acinesia, hipociensia) con afectación apical o sin ella, que se extiende más allá del territorio de una arteria coronaria determinada; ocasionalmente hay una situación estresante asociada

Ausencia de lesiones coronarias en la angiografía o de signos angiográficos de rotura aguda de la placa.

Nuevas alteraciones electrocardiográficas (elevación ST y/ o inversión onda T) 0 discreta elevación de troponina.

Ausencia de feocromocitoma o miocarditis

Es fundamental la realización de una coronariografía de forma precoz para su confirmación diagnóstica y su posterior manejo terapéutico, evitando así el empleo de tratamiento fibrinolítico, dado que la mortalidad por las complicaciones hemorrágicas por su uso es mayor que la del SDAT por sí mismo.

En la coronariografía se verán unas coronarias sin lesiones significativas y disfunción ventricular izquierda con hipocinesia, acinesia o discinesia de los segmentos apicales e hipercontractilidad de los basales, causando la balonización del ventrículo izquierdo durante la sístole (Figura 2). Estas alteraciones pueden mantenerse de una a tres semanas.

Su prevalencia real se desconoce al no exitir una definición unificada de lo que es el SDAT ni de sus criterios diagnósticos básicos. 
En cuanto al tratamiento, en el momento actual es fundamentalmente de soporte incluyendo el uso de betabloqueantes, IECAs, aspirina y diuréticos. La anticoagulación a corto plazo podría estar indicada durante el episodio agudo para evitar las complicaciones secundarias a la formación y liberación de trombos por la turbulencia generada dentro de las cavidades coronarias.

La evolución, aunque habitualmente transcurre sin problemas, puede complicarse por la rotura del ventrículo izquierdo, por lo que este síndrome se ha reconocido recientemente como una causa más de muerte súbita.

\section{Bibliografía}

1. Tsuchihashi K, Ueshima K, Uchida T, Ohmuara N, Jimura K, Owa M, et al. Transient left ventricular apical ballooning without coronary artery stenosis:a novel heart syndrome mimicking acute myocardial infarction. J Am Coll Cardiol 2001;38:11-8.

2. Pereira JR, Segovia J, Oteo JF. Síndrome de discinesia apical transitoriancon una complicación inhabitual. Rev Esp Cardiol 2002;55:1328-32.

3. Barriales Vila R, Bilbao Quesada R, Iglesias Rió E, Bayón Melero N, Mantilla González R, Penas Lado M. Síndrome de discinesia apical transitoria sin lesiones coronarias: importancia del gradiente intraventricular. Rev Esp Cardiol 2004;57:85-8.

4. Ibáñez B, Navarro F, Farré J, Marcos-Alberca P, Orejas M, Rábago R, et al. Asociación del síndrome tako-tsubo con arteria descendente anterior con extensa distribución por el segmento diafragmático. Rev Esp Cardiol 2004;57:2009-16.

5. Abe $\mathrm{Y}$, Kondo M. Apical ballooning of the left ventricle: a distinct entity? Heart 2003;89:974-6

6. Ueyema T, Kasamatsu K, Hano T, Yamamoto K, Tsuruo Y, Nishio I. Emotional stress induces trancient left ventricular hypocontraction in the rat via activation of cardiac adrenoreceptors. a possible animal model of "takotsubo" cardiomyopathy. Circ $\mathrm{J}$ 2002;66:712-3.

7. Segovia Cubero J, Pereira Moral JR. Disfunción apical transitoria: un síndrome en transición hacia la edad adulta. Rev Esp Cardiol 2004;57:194-7.

8. Kurisu S, Sato H, Kawagoe T, Ishihara M, Shimatani Y, Nishioka K, et al. Tako-tsubolike left ventricular dysfunction with ST-segment elevation: a novel cardiac syndrome mimicking acute myocardial infarction. Am Heart J 2002;143:448-55.

9. Wittstein IS, Thiemann DR, Lima JAC, et al. Neurohumoral features of myocardial stunning due to sudden emotional stress. N Engl J Med 2005; 352: 539-48.

10. Laínez B, Ureña M, Álvarez V, Lezaun R. Miocardiopatía de tako-tsubo iatrogénica secundaria a catecolaminas. Rev Esp Cardiol 2009;62(12):1498-508.

11. Sato $H$, Tateishi H, Uchida T. Takotsubo-type cardiomyopathy due to multivessel spasm. En: Kodama K, Haze K, Hon M, eds. Clinical aspect of Myocardial injury: From Ischemia to Heart Failure. Tokyo: Kagakuhyouronsha; 1990. p. 56-64

12. Prasad A, Lerman A, Rihal CS. Apical ballooning syndrome (Tako-Tsubo or stress cardiomyopathy): a mimic of acute myocardial infarction. Am Heart J. 2008; 155:408-17.

13. Núñez Gil IJ, Luaces Méndez M, García-Rubira JC. Cardiopatía de estrés o Síndrome de Tako-tsubo: Conceptos Actuales. Rev Argent Cardiol. 2009; 77:218-23.

14. Akashi YJ, Musha H, Nakazawa K, Miyake F. Plasma brain natriuretic peptide in takotsubo cardiomyopathy. QJM. 2004; 97:599-607.

15. Nef HM, Möllmann H, Elsässer A. Tako-tsubo cardiomyopathy (Apical ballooning). Heart. 2007; 93:1309-15. 